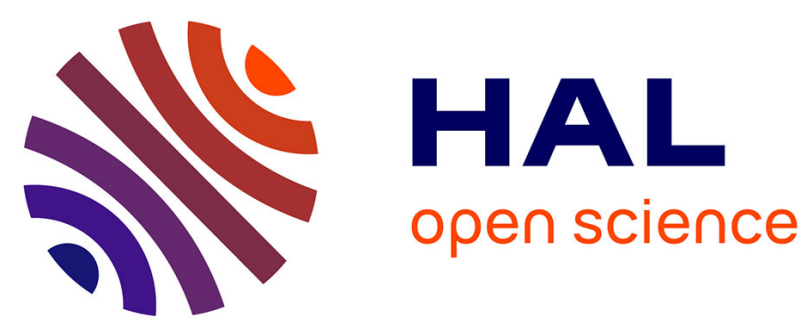

\title{
Generating functions and congruences for 9-regular and 27-regular partitions in 3 colours
}

\author{
Nayandeep Deka Baruah, Hirakjyoti Das
}

\section{To cite this version:}

Nayandeep Deka Baruah, Hirakjyoti Das. Generating functions and congruences for 9-regular and 27-regular partitions in 3 colours. Hardy-Ramanujan Journal, 2022, Special Commemorative volume in honour of Srinivasa Ramanujan - 2021, Volume 44 - Special Commemorative volume in honour of Srinivasa Ramanujan - 2021, pp.101 - 115. 10.46298/hrj.2022.8927 . hal-03498213

\section{HAL Id: hal-03498213 \\ https://hal.science/hal-03498213}

Submitted on 20 Dec 2021

HAL is a multi-disciplinary open access archive for the deposit and dissemination of scientific research documents, whether they are published or not. The documents may come from teaching and research institutions in France or abroad, or from public or private research centers.
L'archive ouverte pluridisciplinaire HAL, est destinée au dépôt et à la diffusion de documents scientifiques de niveau recherche, publiés ou non, émanant des établissements d'enseignement et de recherche français ou étrangers, des laboratoires publics ou privés. 


\title{
Generating functions and congruences for 9-regular and 27-regular partitions in 3 colours
}

\author{
Nayandeep Deka Baruah and Hirakjyoti Das
}

In memory of the 100th anniversary of Srinivasa Ramanujan's eternal rest.

\begin{abstract}
Let $b_{\ell ; 3}(n)$ denote the number of $\ell$-regular partitions of $n$ in 3 colours. In this paper, we find some general generating functions and new infinite families of congruences modulo arbitrary powers of 3 when $\ell \in\{9,27\}$. For instance, for positive integers $n$ and $k$, we have

$$
\begin{aligned}
b_{9 ; 3}\left(3^{k} \cdot n+3^{k}-1\right) & \equiv 0\left(\bmod 3^{2 k}\right), \\
b_{27 ; 3}\left(3^{2 k+3} \cdot n+\frac{3^{2 k+4}-13}{4}\right) & \equiv 0\left(\bmod 3^{2 k+5}\right) .
\end{aligned}
$$
\end{abstract}

Keywords. $\ell$-Regular partitions, Congruences, Generating functions.

2010 Mathematics Subject Classification. 05A17, 11P83

\section{Introduction}

For complex numbers $a_{1}, a_{2}, a_{3}, \ldots, a_{n}$, and $q$ such that $|q|<1$, we define

$$
\left(a_{1}, a_{2}, a_{3}, \ldots, a_{n} ; q\right)_{\infty}:=\prod_{j=0}^{\infty}\left(1-a_{1} q^{j}\right) \cdot\left(1-a_{2} q^{j}\right) \cdot\left(1-a_{3} q^{j}\right) \cdots\left(1-a_{n} q^{j}\right) .
$$

For brevity, we also set $E_{n}:=\left(q^{n} ; q^{n}\right)_{\infty}$.

For a positive integer $\ell>1$, a partition of a positive integer $n$ is called an $\ell$-regular partition of $n$ if there is no part which is a multiple of $\ell$. Suppose that $b_{\ell}(n)$ counts the $\ell$-regular partitions of $n$, then the generating function of $b_{\ell}(n)$ is given by

$$
\sum_{n=0}^{\infty} b_{\ell}(n) q^{n}=\frac{E_{\ell}}{E_{1}}
$$

The divisibility properties of the $\ell$-regular partition function $b_{\ell}(n)$ have been quite widely studied for many values of $\ell$. For example, see $\left[\mathrm{AB} 16, \mathrm{CW} 14, \mathrm{DP} 09, \mathrm{Lin} 15, \mathrm{Web11}\right.$. For more studies on $b_{\ell}(n)$, we refer to Hou et al. [HSZ15] and the references cited there.

Now, let $b_{\ell ; 3}(n)$ denote the number of $\ell$-regular partitions of $n$ where a part can appear in 3 colours. The generating function for $b_{\ell ; 3}(n)$ is given by

$$
\sum_{n=0}^{\infty} b_{\ell ; 3}(n) q^{n}=\frac{E_{\ell}^{3}}{E_{1}^{3}}
$$

The first author was partially supported by Grant no. MTR/2018/000157 of Science \& Engineering Research Board (SERB), DST, Government of India under the MATRICS scheme. The second author was partially supported by Council of Scientific \& Industrial Research (CSIR), Government of India under CSIR-JRF scheme. The authors thank both the funding agencies.

We thank episciences.org for providing open access hosting of the electronic journal Hardy-Ramanujan Journal. 
Recently, Gireesh and Naika [GN19] found an infinite family of congruences for $b_{3 ; 3}(n)$ whereas Saikia and Boruah [SB17] obtained some infinite families of arithmetic identities and congruences modulo 2, 4, 5, and 9 for $b_{\ell ; 3}(n)$ when $\ell \in\{2,3,4,5\}$. Very recently, Chern, Tang, and Xia [CTX20] found an infinite family of congruences for $b_{7 ; 3}(n)$ modulo powers of 7 . In this paper, we find some generating functions for $b_{9 ; 3}(n)$ and $b_{27 ; 3}(n)$ and use those to prove new infinite families of congruences. Using the Rogers-Ramanujan continued fraction, defined by

$$
\mathcal{R}(q):=\frac{q^{1 / 5}}{1}+\frac{q}{1}+\frac{q^{2}}{1}+\frac{q^{3}}{1}+\cdots=q^{1 / 5} \frac{\left(q, q^{4} ; q^{5}\right)_{\infty}}{\left(q^{2}, q^{3} ; q^{5}\right)_{\infty}}, \quad|q|<1,
$$

and two modular equations of degree 7 and 13 , we present some more congruences for $b_{9 ; 3}(n)$ modulo 27. Finally, based on empirical evidences, we pose two conjectural congruences for $b_{3^{k} ; 3}(n)$.

The paper is organised in three sections. Henceforth in this section, we state the main results. In Section 2., we refer to some preliminary lemmas along with their proofs. Finally in Section 3., we prove the following results.

Theorem 1.1. For integers $k \geq 1$ and $n \geq 0$, we have

$$
\sum_{n=0}^{\infty} b_{9 ; 3}\left(3^{k} \cdot n+3^{k}-1\right) q^{n}=\sum_{j=1}^{3^{k-1}} x_{k, j} q^{j-1} \frac{E_{3}^{12 j}}{E_{1}^{12 j}},
$$

where

$$
\begin{aligned}
x_{1} & :=\left(x_{1,1}, x_{1,2}, x_{1,3}, \ldots\right)=(9,0,0, \ldots), \\
x_{k+1} & =x_{k} \cdot A, \\
A & :=\left(a_{i, j}\right)_{i, j \geq 1}, \quad a_{i, j}=m_{4 i, i+j},
\end{aligned}
$$

and

$$
M:=\left(m_{i, j}\right)_{i, j \geq 1}=\left(\begin{array}{cccccccc}
3^{2} & 0 & 0 & 0 & 0 & 0 & 0 & \ldots \\
2 \cdot 3 & 3^{5} & 0 & 0 & 0 & 0 & 0 & \ldots \\
1 & 3^{5} & 3^{8} & 0 & 0 & 0 & 0 & \ldots \\
0 & 2 \cdot 3^{2} \cdot 5 & 2^{2} \cdot 3^{7} & 3^{11} & 0 & 0 & 0 & \ldots \\
0 & 3 \cdot 5 & 2^{2} \cdot 3^{5} \cdot 5 & 3^{10} \cdot 5 & 3^{14} & 0 & 0 & \ldots \\
0 & 1 & 2 \cdot 3^{6} & 3^{9} \cdot 11 & 2 \cdot 3^{14} & 3^{17} & 0 & \ldots \\
\vdots & \vdots & \vdots & \vdots & \vdots & \vdots & \vdots & \ddots
\end{array}\right) .
$$

Corollary 1.2. For integers $k \geq 1, m \geq 1$, and $n \geq 0$, we have

$$
\begin{aligned}
b_{9 ; 3}\left(3^{k} \cdot n+3^{k}-1\right) & \equiv 0\left(\bmod 3^{2 k}\right), \\
b_{9 ; 3}\left(3^{k+1} \cdot n+2 \cdot 3^{k}-1\right) & \equiv 0\left(\bmod 3^{2 k+1}\right), \\
b_{9 ; 3}\left(3^{k+1} \cdot 5^{2 m} \cdot n+3^{k} \cdot 5^{2 m-1} \cdot(3 r+2)-1\right) & \equiv 0\left(\bmod 3^{2 k+1}\right),
\end{aligned}
$$

where $r \in\{0,2,3,4\}$.

Theorem 1.3. For integers $k \geq 0$ and $n \geq 0$, we have

$$
\begin{aligned}
& \sum_{n=0}^{\infty} b_{27 ; 3}\left(3^{2 k+3} \cdot n+\frac{3^{2 k+4}-13}{4}\right) q^{n}=\sum_{j=1}^{\infty} y_{2 k, j} q^{j-1} \frac{E_{3}^{3(4 j-1)}}{E_{1}^{3(4 j-1)}} \\
& \sum_{n=0}^{\infty} b_{27 ; 3}\left(3^{2 k+4} \cdot n+\frac{3^{2 k+4}-13}{4}\right) q^{n}=\sum_{j=1}^{\infty} y_{2 k+1, j} q^{j-1} \frac{E_{3}^{3(4 j-3)}}{E_{1}^{3(4 j-3)}}
\end{aligned}
$$


where the summations on the right hand sides terminate and

$$
\begin{aligned}
& y_{0}:=\left(y_{0,1}, y_{0,2}, y_{0,3}, \ldots\right) \\
&=(90882,1654060905,2356143142059,960546727776771, \\
& 169539908883715971,15487542738423687078,790448381019096370893, \\
& 22759149739354570715472,345654586666447542741231, \\
&2153693963075557766310747,0,0,0, \ldots), \\
& y_{k+1}= \begin{cases}y_{k} \cdot U, & \text { when } k \text { is even, }, \\
y_{k} \cdot V, & \text { when } k \text { is odd, }\end{cases}
\end{aligned}
$$

where

$$
U:=\left(u_{i, j}\right)_{i, j \geq 1}, \quad u_{i, j}=m_{4 i-1, i+j-1}, \quad V:=\left(v_{i, j}\right)_{i, j \geq 1}, \quad v_{i, j}=m_{4 i-3, i+j-1},
$$

where $m_{i, j}$ are given by $M$ defined in (1.3).

Corollary 1.4. For integers $k \geq 0, m \geq 1, n \geq 0$, and $\ell \in\{5,7,11\}$, we have

$$
\begin{aligned}
b_{27 ; 3}\left(3^{2 k+3} \cdot n+\frac{3^{2 k+4}-13}{4}\right) & \equiv 0\left(\bmod 3^{2 k+5}\right), \\
b_{27 ; 3}\left(3^{2 k+4} \cdot n+\frac{7 \cdot 3^{2 k+3}-13}{4}\right) & \equiv 0\left(\bmod 3^{2 k+7}\right), \\
b_{27 ; 3}\left(3^{2 k+4} \cdot n+\frac{11 \cdot 3^{2 k+3}-13}{4}\right) & \equiv 0\left(\bmod 3^{2 k+7}\right), \\
b_{27 ; 3}\left(3^{2 k+5} \cdot n+\frac{5 \cdot 3^{2 k+4}-13}{4}\right) & \equiv 0\left(\bmod 3^{2 k+6}\right), \\
b_{27 ; 3}\left(3^{2 k+5} \cdot \ell^{2 m} \cdot n+3^{2 k+5} \cdot \ell^{2 m-1} \cdot r+\frac{3^{2 k+4} \cdot \ell^{2 m}-13}{4}\right) & \equiv 0\left(\bmod 3^{2 k+6}\right),
\end{aligned}
$$

where $r \in\{1,2,3, \ldots, \ell-1\}$,

$$
b_{27 ; 3}\left(3^{2 k+4} \cdot 7^{2 m} \cdot n+3^{2 k+4} \cdot 7^{2 m-1} \cdot s+\frac{3^{2 k+5} \cdot 7^{2 m}-91}{28}\right) \equiv 0\left(\bmod 3^{2 k+7}\right),
$$

where $s \in\{0,2,3,4,5,6\}$.

Empirical evidences suggest that congruences (1.4), (1.5), (1.7), (1.8), and (1.10) are best possible. Similar results may also be derived for $b_{3^{k} ; 3}(n)$ when $k \geq 4$ but with the cost of some very tedious computations. Now, we present some more congruences for $b_{9 ; 3}(n)$.

Theorem 1.5. For integers $n \geq 0$, we have

$$
\begin{aligned}
b_{9 ; 3}(75 n+r) & \equiv 0(\bmod 27), \quad r \in\{14,29,44,59\}, \\
b_{9 ; 3}\left(3 \cdot 5^{2 k}(n+1)-1\right) & \equiv b_{9 ; 3}(3 n+2)(\bmod 27), \\
b_{9 ; 3}(147 n+21 r+20) & \equiv 2 b_{9 ; 3}(21 n+3 r+2)(\bmod 27), \quad r \in\{0,1,2,3,4,5\}, \\
b_{9 ; 3}(147 n+146) & \equiv 2 b_{9 ; 3}(21 n+20)+2 b_{9 ; 3}(3 n+2)(\bmod 27), \\
b_{9 ; 3}(507 n+39 \cdot r+38) & \equiv 2 b_{9 ; 3}(39 n+3 r+2)(\bmod 27), \quad r \in\{0,1,2, \ldots, 11\}, \\
b_{9 ; 3}(507 n+506) & \equiv 2 b_{9 ; 3}(39 n+38)+2 b_{9 ; 3}(3 n+2)(\bmod 27) .
\end{aligned}
$$

It is likely that results similar to those in Theorem 1.5 exist for $b_{3^{k} ; 3}(n), k \geq 3$. We leave those results for the interested readers. 


\section{Preliminary lemmas}

For a power series $\sum_{n=0}^{\infty} P(n) q^{n}$ and a positive integer $k$, we define the ' $h u f f i n g$ ' operator $H_{k}$ as

$$
H_{k}\left(\sum_{n=0}^{\infty} P(n) q^{n}\right)=\sum_{n=0}^{\infty} P(k n) q^{k n} .
$$

The following lemma contains a recurrent modular equation of degree 3 .

Lemma 2.1. [GN19, (2.10), (2.13), and (2.14)] If $\zeta:=\frac{E_{1}^{3}}{q E_{9}^{3}}$ and $\gamma:=\frac{E_{3}^{12}}{q^{3} E_{9}^{12}}$, then for integers $i \geq 1$, we have

$$
\begin{gathered}
\frac{1}{\zeta^{i}}=\frac{1}{\gamma}\left(\frac{27}{\zeta^{i-1}}+\frac{9}{\zeta^{i-2}}+\frac{1}{\zeta^{i-3}}\right), \\
H_{3}(\zeta)=-3, \quad \text { and } \quad H_{3}\left(\zeta^{2}\right)=9 .
\end{gathered}
$$

Now, we construct the matrix $M$ in (1.3). By (2.19), we have

$$
H_{3}\left(\frac{1}{\zeta^{i}}\right)=\frac{1}{\gamma}\left(H_{3}\left(\frac{27}{\zeta^{i-1}}\right)+H_{3}\left(\frac{9}{\zeta^{i-2}}\right)+H_{3}\left(\frac{1}{\zeta^{i-3}}\right)\right) .
$$

The first four cases of the above identity are as follows.

$$
\begin{aligned}
H_{3}\left(\frac{1}{\zeta}\right) & =\frac{9}{\gamma}, & H_{3}\left(\frac{1}{\zeta^{2}}\right) & =\frac{2 \cdot 3}{\gamma}+\frac{3^{5}}{\gamma^{2}}, \\
H_{3}\left(\frac{1}{\zeta^{3}}\right) & =\frac{1}{\gamma}+\frac{3^{5}}{\gamma}+\frac{3^{8}}{\gamma}, & H_{3}\left(\frac{1}{\zeta^{4}}\right) & =\frac{2 \cdot 3^{2} \cdot 5}{\gamma^{2}}+\frac{2^{2} \cdot 3^{7}}{\gamma^{3}}+\frac{3^{11}}{\gamma^{4}} .
\end{aligned}
$$

Therefore, in general we write that

$$
H_{3}\left(\frac{1}{\zeta^{i}}\right)=\sum_{j=1}^{i} \frac{m_{i, j}}{\gamma^{j}} .
$$

Using $m_{i, j}$ 's from the above identity, we construct the matrix $M$ such that $M=\left(m_{i, j}\right)_{i, j \geq 1}$.

Lemma 2.2. The following properties are satisfied by the entries of $M$ defined in (1.3).

(i) $m_{1,1}=3^{2}$, and $m_{1, j}=0$ for all $j \geq 2$.

(ii) $m_{2,1}=2 \cdot 3, m_{2,2}=3^{5}$, and $m_{2, j}=0$ for all $j \geq 3$.

(iii) $m_{3,1}=1, m_{3,2}=3^{5}, m_{3,3}=3^{8}$, and $m_{3, j}=0$ for all $j \geq 4$.

(iv) $m_{i, j}=0$, for all $j>i$ or $i \geq 3 j+1$.

(v) $m_{i, j}=27 m_{i-1, j-1}+9 m_{i-2, j-1}+m_{i-3, j-1}$ for all $i \geq 4, j \geq 2$.

(vi) $m_{3 i, i}=1$ for all $i \geq 1$.

Proof. The properties (i)-(v) easily follow from (2.20)-(2.23) and property (vi) follows from properties (iii) and (v). 
In the remaining lemmas of this section, we state and prove the main tools of the paper.

Lemma 2.3. For integers $i \geq 1$ and $j \geq 1$, we have

$$
H_{3}\left(q^{i} \frac{E_{3}^{12 i}}{E_{1}^{12 i}}\right)=\sum_{j=1}^{3 i} a_{i, j} q^{3 j} \frac{E_{9}^{12 j}}{E_{3}^{12 j}},
$$

where $a_{i, j}=m_{4 i, i+j}$.

Proof. From property (iv) in Lemma 2.2, it is easy to see that $m_{4 i, j}=0$ when $i \geq j$. Therefore,

$$
H_{3}\left(\frac{1}{\zeta^{4 i}}\right)=\sum_{j=i+1}^{4 i} \frac{m_{4 i, j}}{\gamma^{j}}=\sum_{j=1}^{3 i} \frac{m_{4 i, i+j}}{\gamma^{i+j}},
$$

which can be rewritten as

$$
H_{3}\left(q^{4 i} \frac{E_{9}^{12 i}}{E_{1}^{12 i}}\right)=\sum_{j=1}^{3 i} m_{4 i, i+j} q^{3 i+3 j} \frac{E_{9}^{12 i+12 j}}{E_{3}^{12 i+12 j}},
$$

that gives

$$
H_{3}\left(q^{i} \frac{E_{3}^{12 i}}{E_{1}^{12 i}}\right)=\sum_{j=1}^{3 i} a_{i, j} q^{3 j} \frac{E_{9}^{12 j}}{E_{3}^{12 j}},
$$

where $a_{i, j}=m_{4 i, i+j}$, which is $(2.24)$.

Lemma 2.4. For integers $i \geq 1$ and $j \geq 1$, we have

$$
\begin{aligned}
H_{3}\left(q^{i-3} \frac{E_{3}^{3(4 i-3)}}{E_{1}^{3(4 i-3)}}\right) & =\sum_{j=1}^{3 i-2} u_{i, j} q^{3 j-3} \frac{E_{9}^{3(4 j-1)}}{E_{3}^{3(4 j-1)}}, \\
H_{3}\left(q^{i-1} \frac{E_{9}^{3(4 j-1)}}{E_{3}^{3(4 j-1)}}\right) & =\sum_{j=1}^{3 i} v_{i, j} q^{3 j-3} \frac{E_{9}^{3(4 j-3)}}{E_{3}^{3(4 j-3)}},
\end{aligned}
$$

where $u_{i, j}=m_{4 i-1, i+j-1}$ and $v_{i, j}=m_{4 i-3, i+j-1}$.

Proof. The proofs of the identities in the lemma are similar to that of Lemma 2.3 or one may see in [GN19, (2.21)-(2.26)].

For the next two lemmas of this section, let $\nu(n)$ denote the largest power of 3 that divides $n$.

Lemma 2.5. For integers $k \geq 1$ and $j \geq 1$, we have

$$
\nu\left(x_{k, j}\right) \geq 2 k+\delta_{j, 1}+\left\lfloor\frac{9 j-10}{2}\right\rfloor,
$$

where $x_{k, j}$ are defined in Theorem 1.1 and $\delta_{j, 1}$ is the Kronecker delta function..

Proof. From [Tan19, Lemma 3.2, (3.7)], we recall that

$$
\nu\left(m_{i, j}\right) \geq\left\lfloor\frac{9 j-3 i-1}{2}\right\rfloor
$$


and so,

$$
\nu\left(a_{i, j}\right)=\nu\left(m_{4 i, i+j}\right) \geq\left\lfloor\frac{9 j-3 i-1}{2}\right\rfloor .
$$

In Theorem 1.1, it is defined that $x_{1}=(9,0,0, \ldots)$. So, $\nu\left(x_{1,1}\right)=2$ which is in accordance with (2.25) and since $x_{1, j}=0$ when $j \geq 2,(2.25)$ is true for $k=1$ and $j \geq 1$. We assume that (2.25) is true for an integer $k \geq 1$ and $j \geq 1$. So, we have

$$
\nu\left(x_{k, j}\right) \geq 2 k+\delta_{j, 1}+\left\lfloor\frac{9 j-10}{2}\right\rfloor .
$$

We prove (2.25) dividing $j$ into two cases.

Case 1: When $j=1$,

$$
\nu\left(x_{k+1,1}\right)=\nu\left(\sum_{i=1}^{3^{k-1}} x_{k, i} a_{i, 1}\right) .
$$

Using Lemma 2.2, we have $a_{1,1}=m_{4,2}=90, a_{2,1}=m_{8,3}=24, a_{3,1}=m_{12,4}=1$ and $a_{i, 1}=0$ when $i \geq 4$. Therefore, from the above identity, we have

$$
\begin{aligned}
\nu\left(x_{k+1,1}\right) & \geq \min \left\{\nu\left(x_{k, 1} \cdot a_{1,1}\right), \nu\left(x_{k, 2} \cdot a_{2,1}\right), \nu\left(x_{k, 3} \cdot a_{3,1}\right)\right\} \\
& \geq \min \{2 k+2,2 k+5,2 k+8\}=2(k+1) .
\end{aligned}
$$

Case 2: When $j>1$,

$$
\nu\left(x_{k+1, j}\right)=\nu\left(\sum_{i=1}^{3^{k-1}} x_{k, i} a_{i, j}\right) \geq \min _{1 \leq i \leq 3^{k-1}}\left\{\nu\left(x_{k, i}\right)+\nu\left(a_{i, j}\right)\right\} .
$$

Using (2.27) and (2.28) in the above inequality, we have

$$
\begin{aligned}
\nu\left(x_{k+1, j}\right) & \geq \min _{1 \leq i \leq 3^{k-1}}\left\{2 k+\delta_{i, 1}+\left\lfloor\frac{9 i-10}{2}\right\rfloor+\left\lfloor\frac{9 j-3 i-1}{2}\right\rfloor\right\} \\
& =2 k+\left\lfloor\frac{9 j-4}{2}\right\rfloor>2(k+2)+\left\lfloor\frac{9 j-10}{2}\right\rfloor .
\end{aligned}
$$

Therefore, by (2.29) and (2.30), (2.25) is true for $k+1$ and $j \geq 1$ also. Thus, by induction, (2.25) is true for all $k \geq 1$ and $j \geq 1$.

Lemma 2.6. For integers $k \geq 0$ and $j \geq 1$, we have

$$
\nu\left(y_{2 k, j}\right) \geq 2 k+5+\delta_{j, 1}+\left\lfloor\frac{9 j-10}{2}\right\rfloor
$$

where $y_{k, j}$ are defined in Theorem 1.3.

Proof. By the definition of $y_{0}$ in Theorem 1.3, (2.31) is true for $k=0$ and $j \geq 1$. We assume that (2.31) is true for an integer $k \geq 0$ and $j \geq 1$. Then, we show that (2.31) is true for $k+1$ and $j \geq 1$. Note that due to (2.26),

$$
\begin{aligned}
& \nu\left(u_{i, j}\right)=\nu\left(m_{4 i-1, i+j-1}\right) \geq\left\lfloor\frac{9 j-3 i-7}{2}\right\rfloor, \\
& \nu\left(v_{i, j}\right)=\nu\left(m_{4 i-3, i+j-1}\right) \geq\left\lfloor\frac{9 j-3 i-1}{2}\right\rfloor .
\end{aligned}
$$


We proceed separating $j$ into two cases, when $j=1$ and when $j>1$.

Case 1: When $j=1$,

$$
\nu\left(y_{2 k+1,1}\right)=\nu\left(\sum_{i=1}^{\infty} y_{2 k, i} \cdot u_{i, 1}\right) .
$$

Since $u_{1,1}=1$ and $u_{i, 1}=0$ when $i \geq 2$, we have

$$
\nu\left(y_{2 k+1,1}\right)=\nu\left(y_{2 k, 1}\right) \geq 2 k+5 .
$$

Case 2: When $j>1$,

$$
\begin{aligned}
\nu\left(y_{2 k+1, j}\right) & =\nu\left(\sum_{i=1}^{\infty} y_{2 k, i} \cdot u_{i, j}\right) \\
& \geq \min _{i \geq 1}\left(\nu\left(y_{2 k, i}\right)+\nu\left(u_{i, j}\right)\right) \\
& \geq \min _{i \geq 1}\left(2 k+5+\delta_{i, 1}+\left\lfloor\frac{9 i-10}{2}\right\rfloor+\left\lfloor\frac{9 j-3 i-7}{2}\right\rfloor\right) \\
& =2 k+5+\left\lfloor\frac{9 j-10}{2}\right\rfloor .
\end{aligned}
$$

Therefore, by (2.32) and (2.33), we obtain

$$
\nu\left(y_{2 k+1, j}\right) \geq 2 k+5+\delta_{j, 1}+\left\lfloor\frac{9 j-10}{2}\right\rfloor .
$$

Now, we again proceed with the two cases of $j$, when $j=1$ and when $j>1$.

Case 1: When $j=1$,

$$
\nu\left(y_{2 k+2,1}\right)=\nu\left(\sum_{i=1}^{\infty} y_{2 k+1, i} \cdot v_{i, 1}\right) .
$$

Using Lemma 2.2 and the definition of $v_{i, j}$ it is easy to check that $v_{1,1}=9, v_{2,1}=15, v_{3,1}=1$ and $v_{i, 1}=0$ when $i \geq 4$. Therefore, from the above identity, we have

$$
\begin{aligned}
\nu\left(y_{2 k+2,1}\right) & \geq \min \left\{\nu\left(y_{2 k+1,1} \cdot v_{1,1}\right), \nu\left(y_{2 k+1,2} \cdot v_{2,1}\right), \nu\left(y_{2 k+1,3} \cdot v_{3,1}\right)\right\} \\
& \geq \min \{2 k+7,2 k+10,2 k+13\}=2(k+1)+5 .
\end{aligned}
$$

Case 2: When $j>1$,

$$
\begin{aligned}
\nu\left(y_{2 k+2, j}\right) & =\nu\left(\sum_{i=1}^{\infty} y_{2 k+1, i} \cdot v_{i, j}\right) \\
& \geq \min _{i \geq 1}\left(\nu\left(y_{2 k+1, i}\right)+\nu\left(v_{i, j}\right)\right) \\
& \geq \min _{i \geq 1}\left(2 k+5+\delta_{i, 1}+\left\lfloor\frac{9 i-10}{2}\right\rfloor+\left\lfloor\frac{9 j-3 i-1}{2}\right\rfloor\right) \\
& =2 k+5+\left\lfloor\frac{9 j-4}{2}\right\rfloor>2 k+2+5+\left\lfloor\frac{9 j-10}{2}\right\rfloor .
\end{aligned}
$$

Thus, (2.35) and (2.36) ensure that (2.31) is true for $k+1$ and $j \geq 1$ also. So, by induction, (2.31) is true for all $k \geq 0$ and $j \geq 1$. Thus, the lemma is proved. 
The following lemma states two identities that involve the Rogers-Ramanujan continued fraction.

Lemma 2.7. [Hir17a, p. 85, (8.1.1) and p. 89, (8.4.3)] If $R(q):=\frac{q^{1 / 5}}{\mathcal{R}(q)}$, where $\mathcal{R}(q)$ is defined in (1.1), then

$$
\begin{aligned}
E_{1} & =E_{25}\left(R\left(q^{5}\right)-q-\frac{q^{2}}{R\left(q^{5}\right)}\right), \\
R^{5}(q)-\frac{q^{2}}{R^{5}(q)} & =11 q+\frac{E_{1}^{6}}{E_{5}^{6}} .
\end{aligned}
$$

Lemma 2.8. We have

$$
\begin{gathered}
H_{5}\left(q^{-2} E_{1}^{2}\right)=-E_{5}^{2}, \\
H_{7}\left(q^{-4} E_{1}^{2}\right)=E_{7}^{2}, \\
H_{11}\left(q^{-10} E_{1}^{2}\right)=E_{11}^{2}, \\
H_{7}\left(q^{-5} E_{1}^{6}\right)=49 q E_{7}^{6} .
\end{gathered}
$$

Proof. From [Ber91, p. 274, Theorem 12.1], we recall the following $n$-dissection of $E_{1}$ for $n \equiv$ $\pm 1(\bmod 6)$. Let $n \geq 5$ be an integer such that $n \equiv \pm 1(\bmod 6)$. Let $n=6 m+1$, then, we have

$$
E_{1}=E_{n^{2}} \cdot\left((-1)^{m} q^{\left(n^{2}-1\right) / 24}+\sum_{j=1}^{(n-1) / 2}(-1)^{j+m} q^{(j-m)(3 j-3 m-1) / 2} \frac{\left(q^{2 j n}, q^{n^{2}-2 j n} ; q^{n^{2}}\right)_{\infty}}{\left(q^{j n}, q^{n^{2}-j n} ; q^{n^{2}}\right)_{\infty}}\right) .
$$

Let $n=6 m-1$, then, we have

$$
E_{1}=E_{n^{2}} \cdot\left((-1)^{m} q^{\left(n^{2}-1\right) / 24}+\sum_{j=1}^{(n-1) / 2}(-1)^{j+m} q^{(j-m)(3 j-3 m+1) / 2} \frac{\left(q^{2 j n}, q^{n^{2}-2 j n} ; q^{n^{2}}\right)_{\infty}}{\left(q^{j n}, q^{n^{2}-j n} ; q^{n^{2}}\right)_{\infty}}\right) .
$$

Note that (2.37) is a special case of (2.44) when $n=5$. Raising the 5-, 7-, and 11-dissections of $E_{1}$ obtained from (2.43) and (2.44) to the relevant power, we obtain (2.39)-(2.42) easily.

The next lemma contains a modular equation of degree 7 .

Lemma 2.9. [Hir17a, p. 76] If $\alpha:=\frac{E_{1}}{q^{2} E_{49}}$ and $\beta:=\frac{E_{7}^{4}}{q^{7} E_{49}^{4}}$, then

$$
\alpha^{7}=\beta^{2}-\left(7 \alpha^{3}+35 \alpha^{2}+49 \alpha\right) \beta-7 \alpha^{6}-21 \alpha^{5}-49 \alpha^{4}-147 \alpha^{3}-343 \alpha^{2}-343 \alpha .
$$

From the above lemma, we have

$$
\begin{aligned}
H_{7}\left(\alpha^{n+7}\right)= & \beta^{2} H_{7}\left(\alpha^{n}\right)-\left(7 H_{7}\left(\alpha^{n+3}\right)+35 H_{7}\left(\alpha^{n+2}\right)+49 H_{7}\left(\alpha^{n+1}\right)\right) \beta \\
& -7 H_{7}\left(\alpha^{n+6}\right)-21 H_{7}\left(\alpha^{n+5}\right)-49 H_{7}\left(\alpha^{n+4}\right)-147 H_{7}\left(\alpha^{n+3}\right) \\
& -343 H_{7}\left(\alpha^{n+2}\right)-343 H_{7}\left(\alpha^{n+1}\right)
\end{aligned}
$$

and from [Hir17a, pp. 76-77], we have the the initial cases

$$
\begin{aligned}
& H_{7}(1)=1, \quad H_{7}(\alpha)=-1, \quad H_{7}\left(\alpha^{2}\right)=1, \quad H_{7}\left(\alpha^{3}\right)=-7, \\
& H_{7}\left(\alpha^{4}\right)=-4 \beta-7, \quad H_{7}\left(\alpha^{5}\right)=10 \beta+49, \quad H_{7}\left(\alpha^{6}\right)=49 \text {. }
\end{aligned}
$$


Using (2.45) along with the above initial cases, we find that

$$
H_{7}\left(\alpha^{24}\right)=-1977326743-16744 \frac{E_{7}^{24}}{q^{42} E_{49}^{24}},
$$

which is a key identity for proving Theorem 1.5.

Finally, we have another important modular equation of degree 13 in the following lemma.

Lemma 2.10. [Hir17b, pp. 323-325] If $\epsilon:=\frac{E_{1}}{q_{169}^{E}}$ and $\rho:=\frac{E_{13}^{2}}{q^{13} E_{169}^{2}}$, then

$$
\begin{aligned}
\epsilon^{13}= & 13 \epsilon^{12}-(13 \rho+91) \epsilon^{11}+(169 \rho+507) \epsilon^{10}-\left(78 \rho^{2}+1183 \rho+2535\right) \epsilon^{9} \\
& +\left(962 \rho^{2}+6253 \rho+10985\right) \epsilon^{8}-\left(260 \rho^{3}+6422 \rho^{2}+28561 \rho+41743\right) \epsilon^{7} \\
& +\left(2886 \rho^{3}+31096 \rho^{2}+112047 \rho+142805\right) \epsilon^{6} \\
& -\left(494 \rho^{4}+17238 \rho^{3}+123032 \rho^{2}+371293 \rho+428415\right) \epsilon^{5} \\
& +\left(4498 \rho^{4}+71318 \rho^{3}+404248 \rho^{2}+1056757 \rho+1113879\right) \epsilon^{4} \\
& -\left(468 \rho^{5}+21294 \rho^{4}+224094 \rho^{3}+1085318 \rho^{2}+2599051 \rho+2599051\right) \epsilon^{3} \\
& +\left(2652 \rho^{5}+58474 \rho^{4}+487734 \rho^{3}+2113514 \rho^{2}+4826809 \rho+4826809\right) \epsilon^{2} \\
& -\left(143 \rho^{6}+6084 \rho^{5}+83486 \rho^{4}+571220 \rho^{3}+2227758 \rho^{2}+4826809 \rho+4826809\right) \epsilon+\rho^{7} .
\end{aligned}
$$

Again, from the above lemma, by deriving a recurrence relation similar to the previous one, and using the following initial cases from [Hir17b, pp. 323-325],

$$
\begin{array}{ll}
H_{13}(1)=1, \quad H_{13}(\epsilon)=1, & H_{13}\left(\epsilon^{2}\right)=-2 \rho-1, \quad H_{13}\left(\epsilon^{3}\right)=13, \\
H_{13}\left(\epsilon^{4}\right)=2 \rho^{2}-13, & H_{13}\left(\epsilon^{5}\right)=-20 \rho^{2}-130 \rho-169, \\
H_{13}\left(\epsilon^{6}\right)=10 \rho^{3}-169, & H_{13}\left(\epsilon^{7}\right)=98 \rho^{3}+364 \rho^{2}-169, \\
H_{13}\left(\epsilon^{8}\right)=-70 \rho^{4}-2197, & \\
H_{13}\left(\epsilon^{9}\right)=-162 \rho^{4}+108 \cdot 13 \rho^{3}+72 \cdot 13^{2} \rho^{2}+18 \cdot 13^{3} \rho+13^{4}, \\
H_{13}\left(\epsilon^{10}\right)=238 \rho^{5}-13^{4}, \\
H_{13}\left(\epsilon^{11}\right)=-902 \rho^{5}-1672 \cdot 13 \rho^{4}-792 \cdot 13^{2} \rho^{3}-198 \cdot 13^{3} \rho^{2}-22 \cdot 13^{4} \rho-13^{5}, \\
H_{13}\left(\epsilon^{12}\right)=-418 \rho^{6}-13^{5},
\end{array}
$$

we obtain

$$
H_{13}\left(\epsilon^{24}\right)=-1792160394037-577738 \frac{E_{13}^{24}}{q^{156} E_{169}^{24}},
$$

which is another key identity in order to prove Theorem 1.5.

We are now ready to prove the results of this paper.

\section{Proofs of the main results}

Proof of Theorem 1.1. We have

$$
\sum_{n=0}^{\infty} b_{9 ; 3}(n) q^{n+1}=q \frac{E_{9}^{3}}{E_{1}^{3}}=\frac{1}{\zeta} .
$$


Applying $H_{3}$ in the above, we find that

$$
\sum_{m=1}^{\infty} b_{9 ; 3}(3 m-1) q^{3 m}=H_{3}\left(\frac{1}{\zeta}\right)=\frac{9}{\gamma}=9 q^{3} \frac{E_{9}^{12}}{E_{3}^{12}} .
$$

Since $m \geq 1$, replacing $m$ by $n+1, n \geq 0$, and then replacing $q^{3}$ by $q$, we have

$$
\sum_{n=0}^{\infty} b_{9 ; 3}(3 n+2) q^{n}=9 \frac{E_{3}^{12}}{E_{1}^{12}}
$$

which is the case $k=1$ of (1.2). We prove (1.2) by employing induction on $k$. Suppose that (1.2) is true for an integer $k \geq 1$. Therefore, we have

$$
\sum_{n=0}^{\infty} b_{9 ; 3}\left(3^{k} \cdot n+3^{k}-1\right) q^{n}=\sum_{i=1}^{3^{k-1}} x_{k, i} q^{i-1} \frac{E_{3}^{12 i}}{E_{1}^{12 i}}
$$

which on rearranging gives

$$
\sum_{n=0}^{\infty} b_{9 ; 3}\left(3^{k} \cdot n+3^{k}-1\right) q^{n+1}=\sum_{i=1}^{3^{k-1}} x_{k, i} q^{i} \frac{E_{3}^{12 i}}{E_{1}^{12 i}} .
$$

Extracting the terms involving $q^{3 r}$ for integers $r \geq 1$, from the above, we obtain

$$
\sum_{r=1}^{\infty} b_{9 ; 3}\left(3^{k} \cdot(3 r-1)+3^{k}-1\right) q^{3 r}=\sum_{i=1}^{3^{k-1}} x_{k, i} H_{3}\left(q^{i} \frac{E_{3}^{12 i}}{E_{1}^{12 i}}\right) .
$$

By employing (2.24) in the above, we have

$$
\begin{aligned}
\sum_{r=1}^{\infty} b_{9 ; 3}\left(3^{k} \cdot(3 r-1)+3^{k}-1\right) q^{3 r} & =\sum_{i=1}^{3^{k-1}} x_{k, i} \sum_{j=1}^{3 i} a_{i, j} q^{3 j} \frac{E_{9}^{12 j}}{E_{3}^{12 j}} \\
& =\sum_{j=1}^{3^{k}}\left(\sum_{i=1}^{3^{k-1}} x_{k, i} a_{i, j}\right) q^{3 j} \frac{E_{9}^{12 j}}{E_{3}^{12 j}} \\
& =\sum_{j=1}^{3^{k}} x_{k+1, j} q^{3 j} \frac{E_{9}^{12 j}}{E_{3}^{12 j}} .
\end{aligned}
$$

Replacing $q^{3}$ by $q$ and then replacing $r$ by $n+1, n \geq 0$ in the above, we have

$$
\sum_{n=0}^{\infty} b_{9 ; 3}\left(3^{k+1} \cdot n+3^{k+1}-1\right) q^{n+1}=\sum_{j=1}^{3^{k}} x_{k+1, j} q^{j} \frac{E_{3}^{12 j}}{E_{1}^{12 j}} .
$$

On rearranging the above, we finally obtain

$$
\sum_{n=0}^{\infty} b_{9 ; 3}\left(3^{k+1} \cdot n+3^{k+1}-1\right) q^{n}=\sum_{j=1}^{3^{k}} x_{k+1, j} q^{j-1} \frac{E_{3}^{12 j}}{E_{1}^{12 j}} .
$$

So, (1.2) is true for $k+1$ also. Therefore, by induction (1.2) is true for all $k \geq 1$. 
Proof of Corollary 1.2. By (1.2) and (2.25), the infinite family of congruences (1.4) follows evidently. Now, we prove (1.5) and (1.6). From (1.2), we have

$$
\sum_{n=0}^{\infty} b_{9 ; 3}\left(3^{k} \cdot n+3^{k}-1\right) q^{n} \equiv \sum_{j=1}^{3^{k-1}} x_{k, j} q^{j-1} \frac{E_{3}^{12 j}}{E_{1}^{12 j}}\left(\bmod 3^{2 k+1}\right) .
$$

Due to $(2.25)$, for all $j \geq 2$, we have

$$
\nu\left(x_{k, j}\right)>2 k+1
$$

Therefore, with the aid of the above inequality, (3.47) reduces to

$$
\sum_{n=0}^{\infty} b_{9 ; 3}\left(3^{k} \cdot n+3^{k}-1\right) q^{n} \equiv x_{k, 1} \frac{E_{3}^{12}}{E_{1}^{12}} \equiv x_{k, 1} \frac{E_{3}^{12}}{E_{3}^{4}} \equiv x_{k, 1} E_{3}^{8}\left(\bmod 3^{2 k+1}\right) .
$$

Since, there is no term that involves $q^{3 n+1}$ in the right side of the above identity, (1.5) is obtained extracting the terms involving $q^{3 n+1}$ from both sides of (3.48).

To prove (1.6), we first show that

$$
\sum_{n=0}^{\infty} b_{9 ; 3}\left(3^{k+1} \cdot 5^{2 m-1} \cdot n+2 \cdot 3^{k} \cdot 5^{2 m-1}-1\right) q^{n} \equiv x_{k, 1} q E_{5}^{8}\left(\bmod 3^{2 k+1}\right),
$$

from which (1.6) is evident.

Extracting the terms that involve $q^{3 n}$ from both sides of (3.48), we find that

$$
\sum_{n=0}^{\infty} b_{9 ; 3}\left(3^{k+1} \cdot n+3^{k}-1\right) q^{n} \equiv x_{k, 1} E_{1}^{8}\left(\bmod 3^{2 k+1}\right)
$$

Using (2.37), we obtain

$$
H_{5}\left(q^{-3} E_{1}^{8}\right)=-125 q^{5} f_{25}^{8} .
$$

Multiplying both sides of (3.50) by $q^{-3}$ and then applying the above identity, we find that

$$
\sum_{n=0}^{\infty} b_{9 ; 3}\left(3^{k+1} \cdot(5 n+3)+3^{k}-1\right) q^{n} \equiv-125 x_{k, 1} q E_{5}^{8} \equiv x_{k, 1} q E_{5}^{8}\left(\bmod 3^{2 k+1}\right)
$$

which shows that (3.49) is true for $m=1$. Now, we assume that (3.49) is true for an integer $m \geq 1$. Therefore, extracting the terms involving $q^{5 n+1}$ from both sides of (3.49), we have

$$
\sum_{n=0}^{\infty} b_{9 ; 3}\left(3^{k+1} \cdot 5^{2 m} \cdot n+3^{k} \cdot 5^{2 m}-1\right) q^{n} \equiv x_{k, 1} E_{1}^{8}\left(\bmod 3^{2 k+1}\right) .
$$

Again, multiplying both sides of the above identity by $q^{-3}$ and then extracting the terms that involve $q^{5 n+3}$, we obtain

$$
\sum_{n=0}^{\infty} b_{9 ; 3}\left(3^{k+1} \cdot 5^{2 m+1} \cdot n+2 \cdot 3^{k} \cdot 5^{2 m+1}-1\right) q^{n} \equiv x_{k, 1} q E_{5}^{8}\left(\bmod 3^{2 k+1}\right) .
$$

So, (3.49) is true for $m+1$ also. Thus, by induction (3.49) is true for all $m \geq 1$.

Proof of Theorem 1.3. The proof is similar to the proof of Theorem 1.1 which can be accomplished using Lemma 2.4. Therefore, we avoid the detail. 
Proof of Corollary 1.4. Congruence (1.7) is evident from Theorem 1.3 and Lemma 2.6.

Using the fact that $\nu\left(y_{2 k, j}\right)>2 k+7$ for all $j \geq 2$, which follows from (2.31) and Theorem 1.3, we have

$$
\sum_{n=0}^{\infty} b_{27 ; 3}\left(3^{2 k+3} \cdot n+\frac{3^{2 k+4}-13}{4}\right) q^{n} \equiv y_{2 k, 1} \frac{E_{3}^{9}}{E_{1}^{9}} \equiv y_{2 k, 1} \frac{E_{3}^{9}}{E_{3}^{3}} \equiv y_{2 k, 1} E_{3}^{6}\left(\bmod 3^{2 k+7}\right) .
$$

from which extracting the terms that involve $q^{3 n+1}$ and $q^{3 n+2}$, we obtain (1.8) and (1.9), respectively.

Using (2.34) with similar arguments, we can also find that

$$
\begin{aligned}
\sum_{n=0}^{\infty} b_{27 ; 3}\left(3^{2 k+4} \cdot n+\frac{3^{2 k+4}-13}{4}\right) q^{n} & \equiv y_{2 k+1,1} \frac{E_{3}^{3}}{E_{1}^{3}} \equiv y_{2 k+1,1} \frac{E_{3}^{3}}{E_{3}} \\
& \equiv y_{2 k+1,1} E_{3}^{2}\left(\bmod 3^{2 k+6}\right)
\end{aligned}
$$

which gives (1.10) since there is no term involving $q^{3 n+1}$ in the right side of the above identity.

Proofs of (1.11) and (1.12), which are similar to that of (1.6), can be achieved using (3.52) and (3.51), respectively, and Lemma 2.8 .

Proof of Theorem 1.5. First, we prove (1.13) and (1.14). We have

$$
\sum_{n=0}^{\infty} b_{9 ; 3}(3 n+2) q^{n}=9 \frac{E_{3}^{12}}{E_{1}^{12}} \equiv 9 E_{1}^{24}(\bmod 27) .
$$

Using (2.37) in the above identity, extracting the terms involving $q^{5 n+4}$, dividing both the resulting sides by $q^{4}$, and finally replacing $q^{5}$ by $q$, we find that

$$
\begin{aligned}
\sum_{n=0}^{\infty} b_{9 ; 3}(15 n+14) q^{n} \equiv & 9 E_{5}^{24}\left(4830\left(R^{20}(q)+\frac{q^{8}}{R^{20}(q)}\right)-212520 q\left(R^{15}(q)-\frac{q^{6}}{R^{15}(q)}\right)\right. \\
& +3487260 q^{2}\left(R^{10}(q)+\frac{q^{4}}{R^{10}(q)}\right)-25077360 q^{3}\left(R^{5}(q)-\frac{q^{2}}{R^{5}(q)}\right) \\
& \left.+14903725 q^{4}\right)(\bmod 27) .
\end{aligned}
$$

Invoking (2.38) in the above identity, we have

$$
\begin{aligned}
\sum_{n=0}^{\infty} b_{9 ; 3}(15 n+14) q^{n} & \equiv 43470 E_{1}^{24}-439453125 q^{4} E_{5}^{24}(\bmod 27) \\
& \equiv 9 q^{4} E_{5}^{24}(\bmod 27) .
\end{aligned}
$$

Extracting the terms that involve $q^{5 n+r}, r \in\{0,1,2,3\}$ from the above identity, we readily obtain (1.13). On the other hand, extraction of the terms involving $q^{5 n+4}$ from the above will give rise to the following recurrence

$$
b_{9 ; 3}(75 n+74) \equiv b_{9 ; 3}(3 n+2)(\bmod 27),
$$

which by induction implies (1.14).

Now, we prove the remaining congruences. Proofs of (1.15) and (1.16) and that of (1.17) and (1.18) are similar. Therefore, we prove (1.15) and (1.16) only. We have

$$
\sum_{n=0}^{\infty} b_{9 ; 3}(3 n+2) q^{n+1}=9 q \frac{E_{3}^{12}}{E_{1}^{12}} \equiv 9 q E_{1}^{24} \equiv 9 q^{49} E_{49}^{24} \alpha^{24}(\bmod 27) .
$$


Applying $H_{7}$ and then using (2.46) in the above identity, we find that

$$
\begin{aligned}
\sum_{m=1}^{\infty} b_{9 ; 3}(3(7 m-1)+2) q^{7 m} & \equiv 9 q^{49} E_{49}^{24} H_{7}\left(\alpha^{24}\right) \\
& \equiv 18\left(q^{7} E_{7}^{24}+q^{49} E_{49}^{24}\right)(\bmod 27) .
\end{aligned}
$$

Since $m \geq 1$, replacing $m$ by $n+1, n \geq 0$, and then replacing $q^{7}$ by $q$, we have

$$
\sum_{n=0}^{\infty} b_{9 ; 3}(3(7 n+6)+2) q^{n} \equiv 18\left(E_{1}^{24}+q^{6} E_{7}^{24}\right)(\bmod 27),
$$

which can be written as

$$
\sum_{n=0}^{\infty} b_{9 ; 3}(21 n+20) q^{n} \equiv 2 \sum_{n=0}^{\infty} b_{9 ; 3}(3 n+2) q^{n}+2 \sum_{n=0}^{\infty} b_{9 ; 3}(3 n+2) q^{7 n+6}(\bmod 27) .
$$

Extracting the terms involving $q^{7 n+r}, r \in\{0,1,2,3,4,5\}$ and $q^{7 n+6}$ from the above, we deduce (1.15) and (1.16), respectively.

\section{Concluding remarks}

Based on computational evidences, we present the following conjectures.

Conjecture 4.1. If $k \geq 1$ is an even integer, then for integers $\ell \geq 0$ and $n \geq 0$, we have

$$
b_{3^{k} ; 3}\left(3^{\ell+k-1} \cdot n+3^{\ell+k-1}-\frac{3^{k}-1}{8}\right) \equiv 0\left(\bmod 3^{3 k / 2+2 \ell-1}\right) .
$$

Conjecture 4.2. If $k \geq 1$ is an odd integer, then for integers $\ell \geq 0$ and $n \geq 0$, we have

$$
b_{3^{k} ; 3}\left(3^{2 \ell+k} \cdot n+\frac{2 \cdot 3^{2 \ell+k+1}-3^{k}+1}{8}\right) \equiv 0\left(\bmod 3^{3(k-1) / 2+2 \ell+2}\right) .
$$

Acknowledgement. The authors thank the anonymous referee who pointed out some mistakes in the earlier version of the paper.

\section{References}

[AB16] Z. Ahmed and N.D. Baruah, New congruences for $\ell$-regular partitions for $\ell \in\{5,6,7,49\}$, Ramanujan J. 40 (2016), no. $3,649-668$.

[Ber91] B.C. Berndt, Ramanujan's Notebooks. Part III, Springer-Verlag, New York, 1991.

[CTX20] S. Chern, D. Tang, and E.X.W. Xia, Arithmetic properties for 7-regular partition triples, Indian J. Pure Appl. Math. 51 (2020), no. 2, 717-733.

[CW14] R. Carlson and J.J. Webb, Infinite families of infinite families of congruences for k-regular partitions, Ramanujan J. 33 (2014), no. 3, 329-337.

[DP09] B. Dandurand and D. Penniston, $\ell$-divisibility of $\ell$-regular partition functions, Ramanujan J. 19 (2009), no. 1, 63-70.

[GN19] D.S. Gireesh and M.S.M. Naika, On 3-regular partitions in 3-colors, Indian J. Pure Appl. Math. 50 (2019), no. 1, 137-148.

[Hir17a] M.D. Hirschhorn, The Power of q, Developments in Mathematics, vol. 56, Springer, Berlin, 2017.

[Hir17b] M.D. Hirschhorn, Ramanujan's tau function, Analytic number theory, modular forms and q-hypergeometric series, Springer Proc. Math. Stat., vol. 221, Springer, Cham, 2017, pp. 311-328.

[HSZ15] Q.-H. Hou, L.H. Sun, and L. Zhang, Quadratic forms and congruences for $\ell$-regular partitions modulo 3, 5 and 7, Adv. in Appl. Math. 70 (2015), 32-44. 
[Lin15] B.L.S. Lin, Arithmetic of the 7-regular bipartition function modulo 3, Ramanujan J. 37 (2015), no. 3, $469-478$.

[SB17] N. Saikia and C. Boruah, Congruences of $\ell$-regular partition triples for $\ell \in\{2,3,4,5\}$, Acta Math. Vietnam. 42 (2017), no. 3, 551-561.

[Tan19] D. Tang, Congruences modulo powers of 3 for 2-color partition triples, Period. Math. Hungar. 78 (2019), no. 2, $254-266$.

[Web11] J.J. Webb, Arithmetic of the 13-regular partition function modulo 3, Ramanujan J. 25 (2011), no. 1, 49-56

\section{Nayandeep Deka Baruah}

Department of Mathematical Sciences

Tezpur University, Napaam 784028, Assam, India

e-mail: nayan@tezu.ernet.in

\section{Hirakjyoti Das}

Department of Mathematical Sciences

Tezpur University, Napaam 784028, Assam, India

e-mail: hdas@tezu.ernet.in 